\title{
Elevated De Ritis Ratio Is Associated With Poor Prognosis in COVID-19: A Systematic Review and Meta-Analysis
}

OPEN ACCESS

Edited by:

Hu Zhang,

Sichuan University, China

Reviewed by:

Alejandro Piscoya,

Saint Ignatius of Loyola

University, Peru

Giordano Madeddu,

University of Sassari, Italy

*Correspondence:

Raymond Pranata

raymond_pranata@hotmail.com

tORCID:

Raymond Pranata orcid.org/0000-0003-3998-6551

lan Huang

orcid.org/0000-0003-1189-8453

Michael Anthonius Lim orcid.org/0000-0001-7631-6835

Emir Yonas

orcid.org/0000-0002-8604-405X

Sally Aman Nasution orcid.org/0000-0002-0173-049X

Bambang Budi Siswanto orcid.org/0000-0003-3998-1590

Specialty section

This article was submitted to Gastroenterology,

a section of the journal

Frontiers in Medicine

Received: 05 March 2021 Accepted: 22 November 2021 Published: 22 December 2021

Citation:

Pranata $R$, Huang I, Lim MA, Yonas E, Vania $R$, Lukito $A A$, Nasution SA, Siswanto BB and Kuswardhani RAT (2021) Elevated De Ritis Ratio Is

Associated With Poor Prognosis in COVID-19: A Systematic Review and Meta-Analysis. Front. Med. 8:676581.

doi: 10.3389/fmed.2021.676581

\begin{abstract}
Raymond Pranata ${ }^{1 * t}$, Ian Huang ${ }^{1,2+}$, Michael Anthonius Lim ${ }^{1 \dagger}$, Emir Yonas ${ }^{3+}$, Rachel Vania ${ }^{1,4}$, Antonia Anna Lukito ${ }^{1,5}$, Sally Aman Nasution ${ }^{6 \dagger}$, Bambang Budi Siswanto ${ }^{7 \dagger}$ and Raden A. Tuty Kuswardhani ${ }^{8}$

${ }^{1}$ Faculty of Medicine, Universitas Pelita Harapan, Tangerang, Indonesia, ${ }^{2}$ Faculty of Medicine, Department of Internal Medicine, Hasan Sadikin General Hospital, Universitas Padjadjaran, Bandung, Indonesia, ${ }^{3}$ Faculty of Medicine, Universitas YARSI, Jakarta, Indonesia, ${ }^{4}$ Faculty of Medicine, Division of Plastic, Reconstructive, and Aesthetic, Department of Surgery, Sanglah General Hospital, Udayana University, Jimbaran, Indonesia, ${ }^{5}$ Department of Cardiology and Vascular Medicine, Siloam Hospitals Lippo Village, Tangerang, Indonesia, ${ }^{6}$ Faculty of Medicine, Division of Cardiology, Department of Internal Medicine, Universitas Indonesia/Cipto Mangunkusumo National General Hospital, Jakarta, Indonesia, ${ }^{7}$ Faculty of Medicine, Department of Cardiology and Vascular Medicine, National Cardiovascular Center Harapan Kita, Universitas Indonesia, Jakarta, Indonesia, ${ }^{8}$ Faculty of Medicine, Division of Geriatrics, Department of Internal Medicine, Sanglah Teaching Hospital, Udayana University, Denpasar, Indonesia
\end{abstract}

Objective: This meta-analysis aims to assess whether elevated De Ritis ratio is associated with poor prognosis in patients with coronavirus 2019 (COVID-19).

Methods: A systematic literature search was performed using PubMed, Embase, and EuropePMC databases up until September 17, 2021. De Ritis ratio is also known as Aspartate aminotransferase/alanine transaminase (AST/ALT) ratio. The main outcome was poor prognosis, a composite of mortality, severity, the need for ICU care, and intubation. The effect measure was odds ratios (ORs) and mean differences. We generated sensitivity and specificity, negative and positive likelihood ratio (NLR and PLR), diagnostic odds ratio (DOR), and area under curve (AUC).

Results: There were eight studies with 4,606 patients. De Ritis ratio was elevated in $44 \%$ of the patients. Patients with poor prognosis have higher De Ritis ratio [mean difference $0.41(0.31,0.50), p<0.001 ; R^{2}: 81.0 \%$ ] and subgroup analysis showed that non-survivors also have higher De Ritis Ratio [mean difference 0.47 (0.46, 0.48$), p<$ $0.001 ; R^{2}: 0 \%$ ]. Elevated De Ritis ratio was associated with poor prognosis [OR 3.28 $\left.(2.39,4.52), p<0.001 ; I^{2}: 35.8 \%\right]$. It has a sensitivity of $55 \%$ (36-73), specificity of $71 \%$ (52-85), PLR 1.9, NLR.63, DOR of 3 (2-4), and AUC of.67 (0.63-0.71). The posterior probability of poor prognosis was $38 \%$ if De Ritis is elevated, while $17 \%$ if De Ritis is not elevated.

Conclusion: Elevated De Ritis ratio is associated with poor prognosis in patients with COVID-19.

Systematic Review Registration: PROSPERO ID: CRD42020216634.

Keywords: coronavirus - COVID-19, liver enzyme, transaminase, SARS-CoV-2, De Ritis ratio 


\section{INTRODUCTION}

The severe acute respiratory syndrome coronavirus 2 (SARSCoV-2) spread rapidly and causes a considerable number of deaths worldwide (1). Although most patients with coronavirus 2019 disease (COVID-19) have mild-to-moderate symptoms, they may develop severe COVID-19 with multi-organ dysfunction, cardiorespiratory collapse, coagulopathy and thrombosis, sepsis, and even death $(2,3)$. Common symptoms include fever, cough and dyspnea, and minor symptoms are dysgeusia, anosmia, gastrointestinal symptoms, cutaneous manifestation, and headache (4-6). Although the virus primarily affects the lungs, it may invade and damage other organs, such as the heart and vasculature, coagulation system, liver, kidneys, intestine, and central nervous system (7-12).

Severe acute respiratory syndrome coronavirus 2 (SARS-CoV2) has been reported to cause a varying degree of liver injury (13). Liver injury is more frequently found in patients with severe COVID-19 and is associated with an increased risk of poor outcomes (14). The ratio between the two most routinely requested liver function panel, the aspartate aminotransferase (AST)/alanine aminotransferase (ALT) ratio or more commonly known as the De Ritis ratio, was recently reported as a possible biomarker for prognostication in patients with COVID-19 (15). Therefore, we conducted a systematic review and meta-analysis to evaluate the association between De Ritis ratio and composite poor outcomes in COVID-19.

\section{MATERIALS AND METHODS}

The study was registered in the PROSPERO database (CRD42020216634) and was conducted per the Preferred Reporting Items for Systematic Reviews and Meta-Analyses (PRISMA) guidelines.

\section{Eligibility Criteria}

Research articles (both prospective and retrospective cohorts) that contain information on De Ritis ratio and mortality, severity, intensive care unit (ICU) care admission or need for intubation were included in the study. We excluded preprints, review articles, editorial, commentaries, conference abstracts, letters, and case reports/series.

\section{Search Strategy and Study Selection}

We performed a systematic literature search from PubMed database, Embase database, and EuropePMC database with the search terms "COVID-19" OR "2019-nCoV" OR "SARS-CoV2" AND "De Ritis Ratio" OR "AST ALT Ratio." The search was finalized on September 17, 2021. The PubMed search strategy was [(COVID-19) OR (2019-nCoV) OR (SARS-CoV-2)] AND [(De Ritis Ratio) OR (AST ALT Ratio)]. Two independent authors performed the initial search and duplicate removal. The inclusion

Abbreviations: ACE2, Angiotensin receptor enzyme 2; AST, Aspartate aminotransferase; ALT, Alanine aminotransferase; AUC, Area under curve; COVID-19, Coronavirus disease 2019; DOR, Diagnostic odds ratio; OR, Odds ratio; PLR, Positive likelihood ratio; NLR, Negative likelihood ratio; SARS-CoV-2, Severe cute respiratory syndrome coronavirus 2. and exclusion criteria served as the basis for article exclusion during the title or abstract screening and evaluation of fulltext articles.

\section{Data Collection}

Data extraction from the eligible studies was conducted by two authors who are independently using pre-built forms containing the author, study design, origin, AST, ALT, cut-off for elevated De Ritis ratio, sample size, age, gender, obesity, diabetes, elevated liver enzymes, and outcome of interests.

The main outcome was poor prognosis, a composite of mortality, severity, need for ICU care, and need for intubation. Mortality was defined as non-survivor or death.

Severity was defined according to the studies inclusion parameters, need for ICU care, and intubation. The effect measure was the odds ratios (ORs) and mean differences. Diagnostic meta-analysis was performed to generate diagnostic values, which consisted of sensitivity, specificity, negative and positive likelihood ratio (NLR and PLR), diagnostic odds ratio (DOR), and area under curve (AUC).

\section{Risk of Bias Assessment}

The risk of bias assessment was performed independently by two authors with the help of Newcastle-Ottawa Scale (NOS). Discrepancies were resolved by discussion. The Egger's test and Deek's funnel plot asymmetry test was used to assess the presence of small-study effects and publication bias, respectively.

\section{Statistical Analysis}

STATA 16 (College Station, TX) was used to perform statistical analysis. Meta-analysis of proportions was performed to pool the incidence of elevated De Ritis Ratio. DerSimonian and Laird method random-effects models were used to pool ORs and mean differences, notwithstanding heterogeneity. $p<0.05$ were considered statistically significant. Inter-study heterogeneity was evaluated using the I-squared $\left(I^{2}\right)$ and Cochrane $Q$ test, an $I^{2}>50 \%$ or $p<0.10$ indicates substantial heterogeneity. We performed pooling of sensitivity and specificity and generated a summary receiver operating characteristic (SROC) curve. Relationship between prior probability and posterior probability was evaluated using Fagan's nomogram. Subgroup analysis was performed for mortality outcome.

\section{RESULTS}

\section{Baseline Characteristics}

There were eight studies with 4,606 patients in this meta-analysis (Figure 1) (7, 16-19). The mean age of patients in this study was 64.3 years, whereas $46.3 \%$ of the patients were male. The characteristics of the studies are presented in Table 1. Patients with poor prognosis have higher AST levels [mean difference 8.82 (5.47, 12.17), $p<0.001 ; I^{2}: 71.7 \%, p=0.007$ ] (Figure 2A), but not ALT levels [mean difference $0.43(-5.03,5.88), p=0.878 ; I^{2}$ : $88.3 \%, p<0.001$ ] (Figure 2B). De Ritis ratio was elevated in 24\% of the patients. Poor prognosis occurs in $26 \%$ of the patients. 


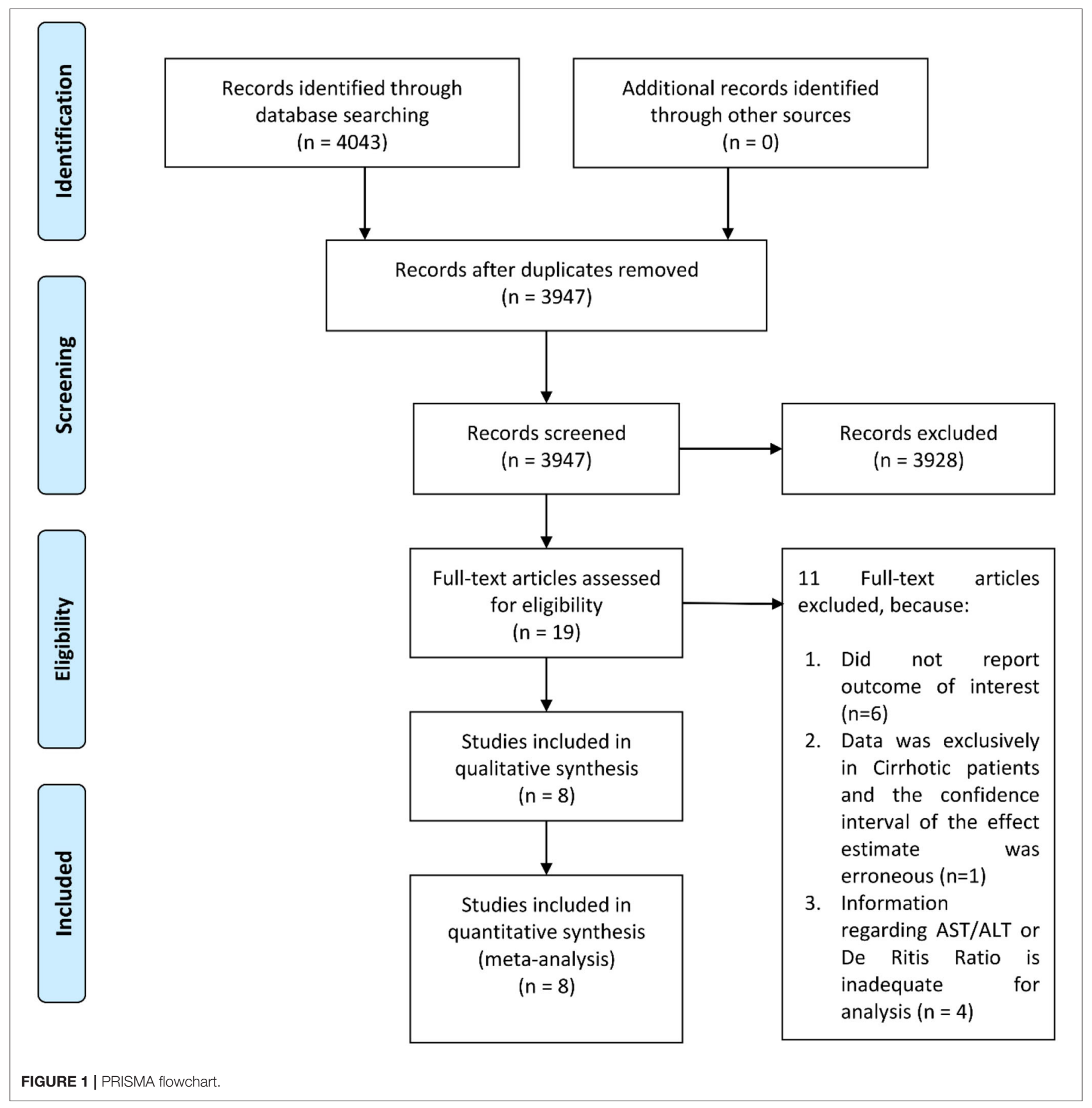

Elevated De Ritis Ratio and Poor Prognosis

Patients with poor prognosis have higher De Ritis ratio [mean difference $\left.0.41(0.31,0.50), p<0.001 ; I^{2}: 81.0 \%, p<0.001\right]$ (Figure 3 ) and subgroup analysis showed that non-survivors also have higher De Ritis Ratio [mean difference $0.47(0.46,0.48), p<$ $\left.0.001 ; I^{2}: 0 \%, p=0.463\right]$. Elevated De Ritis ratio was associated with poor prognosis [OR $3.28(2.39,4.52), p<0.001 ; I^{2}: 35.8 \%$, $p=0.182]$ (Figure 4) and subgroup analysis also showed that elevated De Ritis ratio was associated with mortality [OR 3.36 $\left.(1.93,5.85), p<0.001 ; I^{2}: 51.7 \%, p=0.102\right]$. It has a sensitivity of
$55 \%$ (36-73), specificity of 71\% (52-85), PLR 1.9, NLR 0.63, DOR of 3 (2-4), and AUC of 0.67 (0.63-0.71) (Figure 5). The posterior probability of poor prognosis was $38 \%$ if De Ritis was elevated, while $17 \%$ if De Ritis was not elevated (Figure 6).

\section{Risk of Bias Assessment}

Newcastle-Ottawa Scale (NOS) indicates a low-moderate risk of bias (Table 1). There is no indication of small-study effects in the relationship between elevated De Ritis ratio and poor 


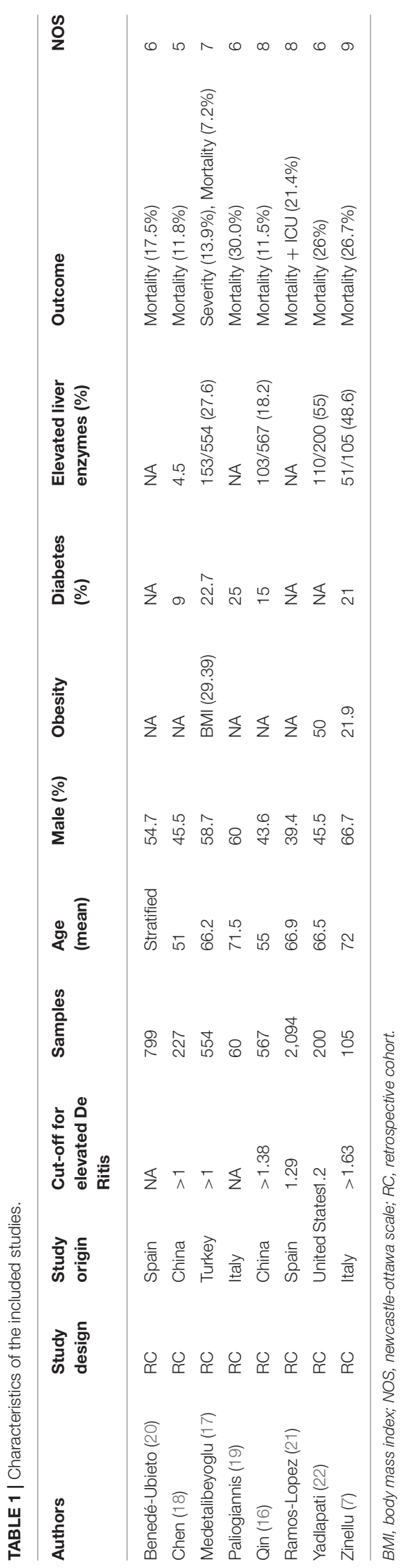

prognosis $(p=0.488)$. Deek's funnel plot asymmetry test was non-significant $(p=0.81)$.

\section{DISCUSSION}

Early identification of patients at risk for developing severe COVID-19 is crucial during the pandemic. Previous studies highlighted that individuals with advanced age, high body mass index, and physical inactivity had greater morbidity and mortality from COVID-19, along with the presence of various comorbidities, such as cardiovascular disease, diabetes, chronic obstructive pulmonary disease, hypertension, and chronic kidney disease (23-31). Several inflammatory parameters, comprising C-reactive protein, D-dimer, procalcitonin, interleukin-6, and ferritin, are often higher in patients with severe and critically ill with COVID-19 (8). An increase in liver-related biomarkers, particularly AST, ALT, total bilirubin concentrations, and gamma-glutamyl transferase in patients with COVID-19 have been reported $(32,33)$.

Although hepatic damage is not commonly seen as a major characteristic of COVID-19, liver injury is an emerging concern because it may indicate a severe disease course (2). The mechanism for liver involvement in COVID-19 remains obscure. Previous liver pathology reports showed the presence of moderate microvesicular steatosis along with mild inflammation in several areas (34). These patterns are also observed in druginduced liver injury and sepsis, although these findings are not unique, they might provide insight into the mechanism involved in liver injury induced by COVID-19 (35). The SARSCoV-2 may invade the liver directly through the angiotensin receptor enzyme 2 (ACE2) receptor, which serves as the novel coronavirus' entry point. It has been found that bile duct epithelial cells (cholangiocytes) express a high amount of ACE2 receptors (36). Liver dysfunction may also be caused by druginduced liver injury or an overactive inflammatory response, including cytokine storm and pneumonia-associated hypoxia (2, 7). Antivirals used in the treatment of COVID-19 are postulated to cause drug-induced liver injury (37).

Serum concentrations of ALT and AST, without exception, are the most frequently ordered liver panel for evaluating liver injury in all laboratories. ALT is present in the cytosol of hepatocytes, while AST is present in the cytoplasm and mitochondria of the hepatocyte (38). ALT activity in the liver is $\sim 10$-fold higher than that of the heart and skeletal muscles, which emphasizes its function to indicate parenchymal liver disease or injury. Meanwhile, AST has the greatest activity in the liver, cardiac, and skeletal muscle, but also exhibits in other tissues including kidneys, pulmonary, brain, pancreas, red blood cells, and white blood cells. Therefore, ALT is a more specific biomarker for liver damage compared to AST, indicating liver-biliary disease, myocardial injury, and rhabdomyolysis $(7,15)$. AST and ALT are found in the liver with a 2.5:1 ratio but with different turnaround time, resulting in a relatively similar level of serum of AST and ALT in healthy populations (38).

The De Ritis ratio or the AST/ALT ratio is a promising biochemical parameter for prognostication in COVID-19. In the 
A

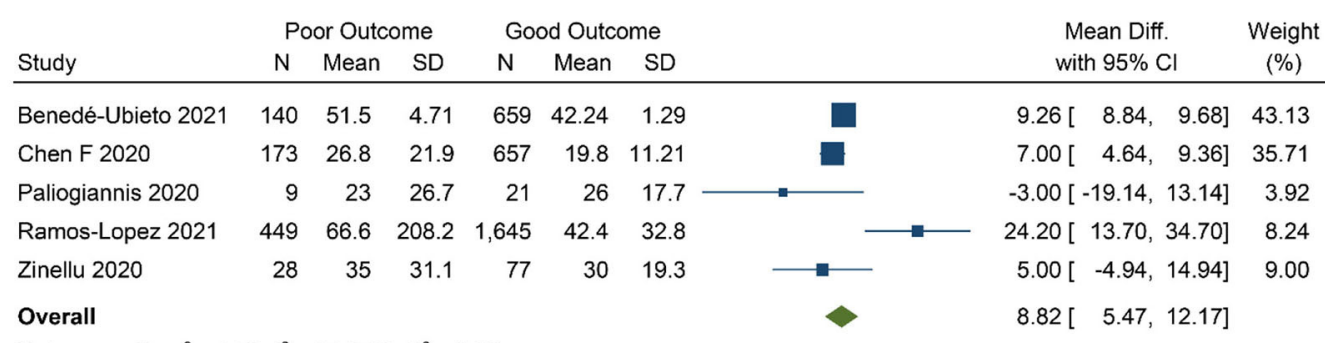

Heterogeneity: $\tau^{2}=6.73, \mathrm{I}^{2}=71.67 \%, \mathrm{H}^{2}=3.53$

Test of $\theta_{i}=\theta_{j}: Q(4)=14.12, p=0.01$

Test of $\theta=0: z=5.16, p=0.00$

Random-effects DerSimonian-Laird model

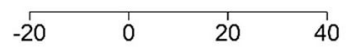

B

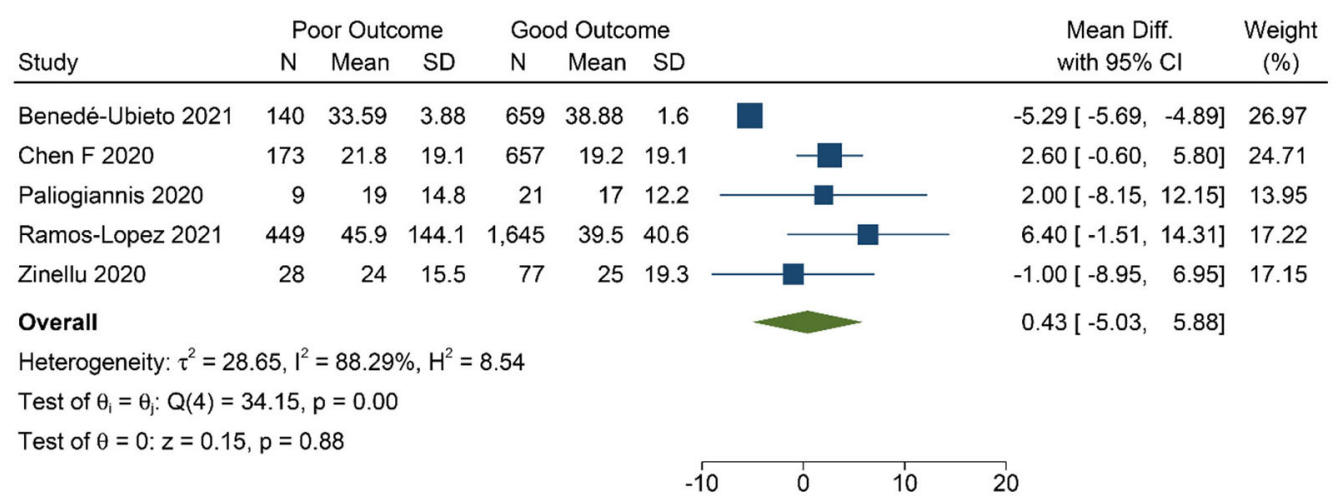

Random-effects DerSimonian-Laird model

FIGURE 2 | Mean difference in aspartate aminotransferase (AST) (A) and alanine transaminase (ALT) (B) level between poor and good prognosis.

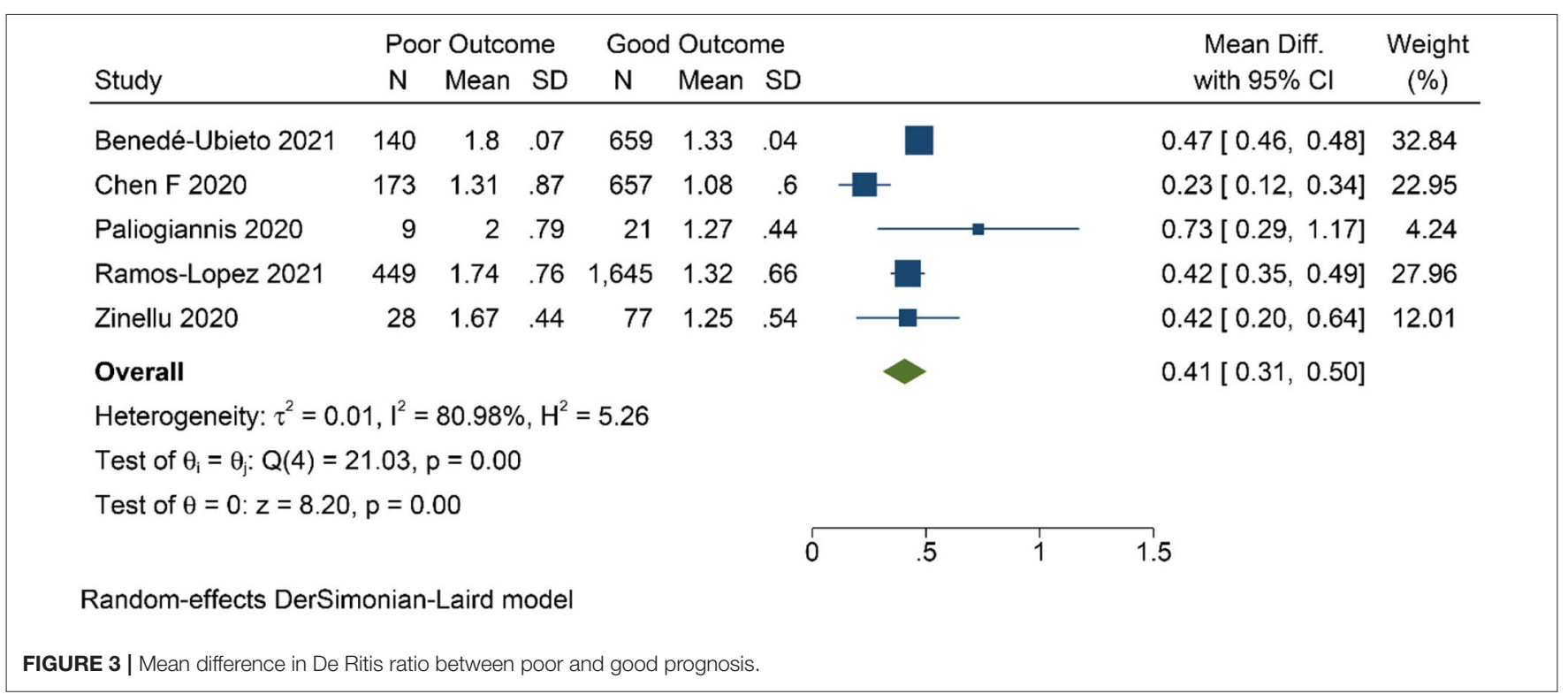




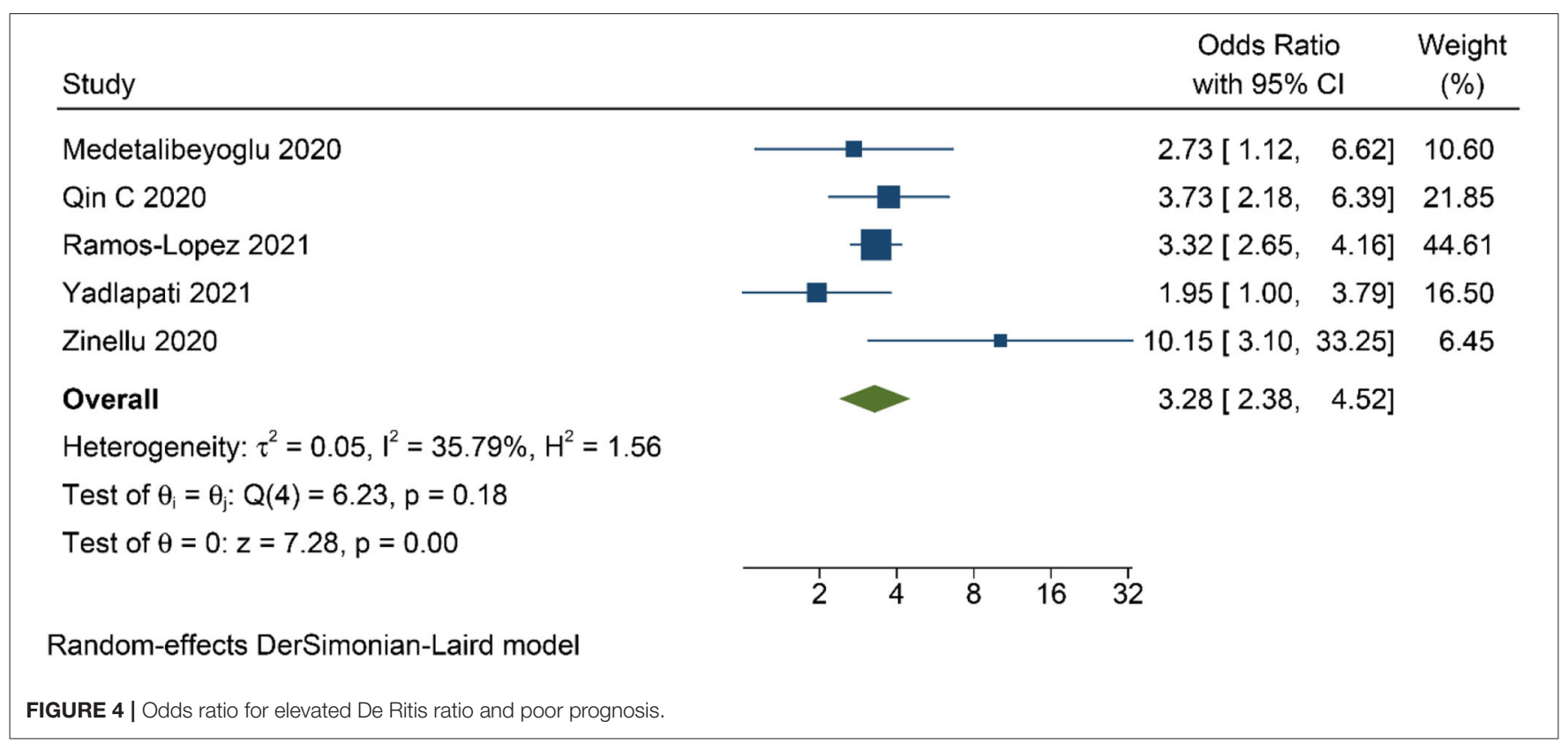

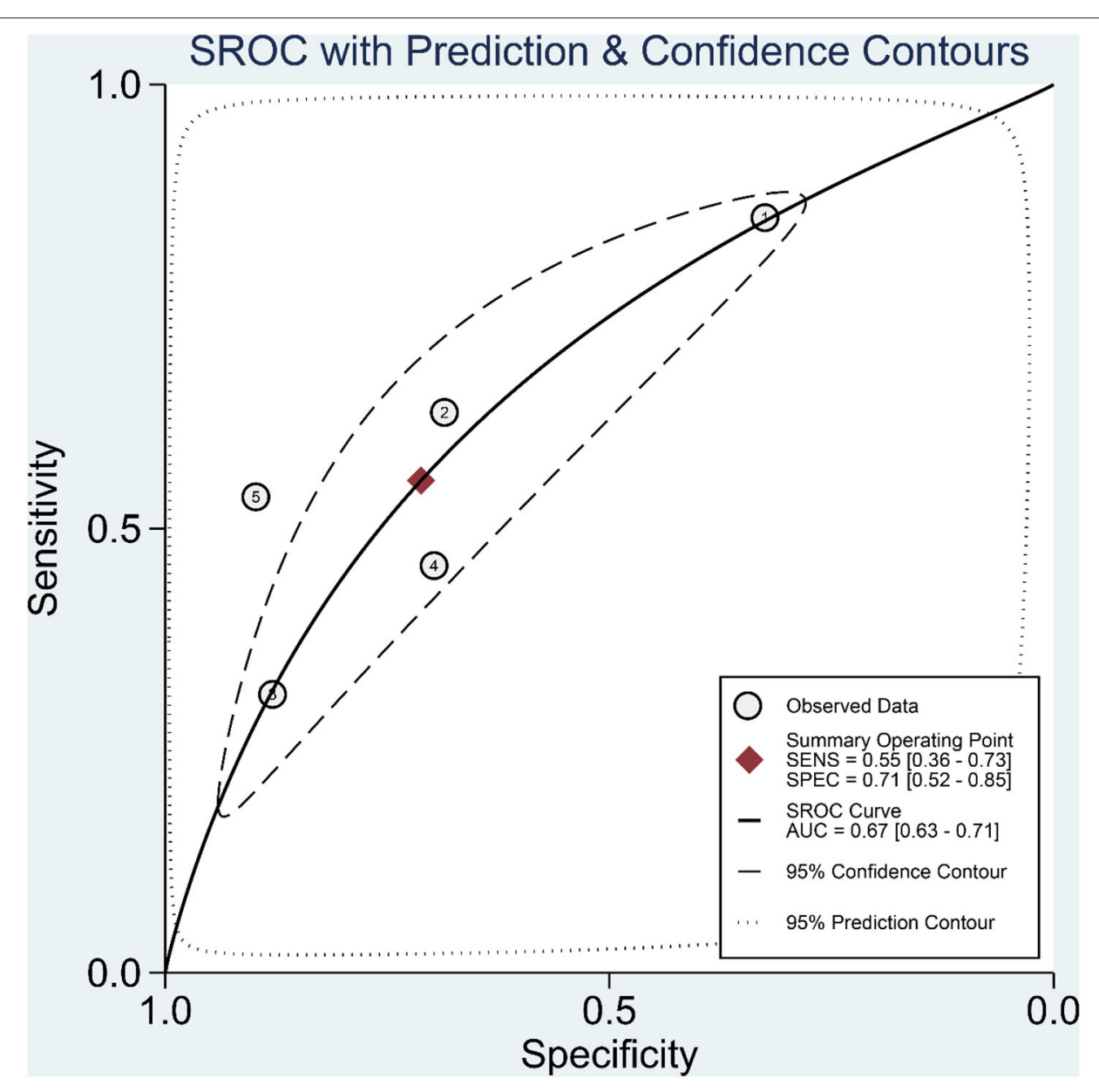

FIGURE 5 | Summary receiver operating characteristic (SROC) curve. 


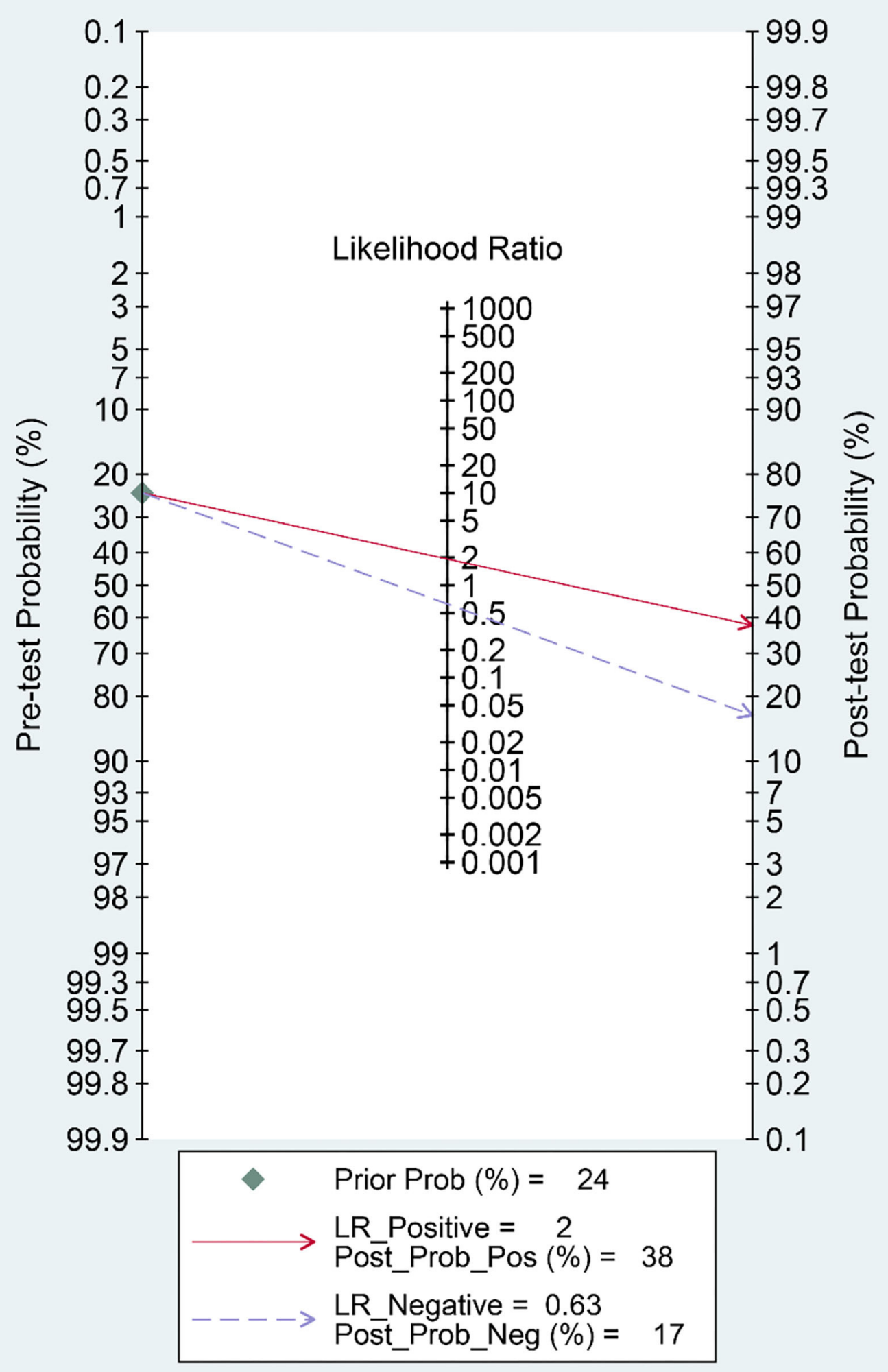


present study, elevated De Ritis ratio was associated with 3fold increased risk for poor prognosis in patients with COVID19. Although the cut-off values for elevated De Ritis ratio are different from these five studies (Table 1), the result of this meta-analysis has low heterogeneity $\left(I^{2}: 35.8 \%\right)$. Nonetheless, the difference in the cut-off value used between those studies caused a highly varied diagnostic value (Figure 3) with an overall sensitivity of $55 \%$, specificity of $71 \%$, and AUC of 0.67 . These variations further translate into the uncertainty of the optimal cut-off value for De Ritis Ratio as a prognostic factor in COVID19 and merit further investigations.

Interestingly, Qin et al. indicated that De Ritis ratio of $\geq 1.38$ was independently associated with poor prognosis irrespective of AST elevation ( $\leq 40$ and $>40 \mathrm{U} / \mathrm{L})$ (16). They showed that AST/ALT ratio elevation was associated with a more severely computed tomography scan findings, higher severity, and positive linear association with other prognostic markers (e.g., c-reactive protein, procalcitonin, interleukin-6, D-Dimer, lactate, $\mathrm{LDH}$, and creatine Kinase-MB). Additionally, Chen et al. showed the association of AST/ALT ratio with liver injury and severity of COVID-19. However, the number of outcomes or risk estimates (e.g., OR) of this study interest was not available (18).

There were two studies on the association of De Ritis ratio with other specific biochemical parameters (e.g., creatinine kinase and serum ALT), but were excluded from the analysis due to its irrelevance with our outcome of interest $(15,39)$.

The limitations of the current study were primarily caused by the small quantity of the included studies. Moreover, the retrospective-observational nature and the small sample size of the included studies should be taken into account in extrapolating the results of this metaanalysis, where selection bias and confounding factors may be evident. We also could not dismiss the possibility of publication bias due to the small number of studies. Despite our limitations, this meta-analysis has brought early

\section{REFERENCES}

1. Livingston E, Bucher K, Rekito A. Coronavirus Disease 2019 and Influenza 2019-2020. JAMA. (2020) 323:1122. doi: 10.1001/jama.2020.2633

2. Lim MA, Pranata R, Huang I, Yonas E, Soeroto AY, Supriyadi R. multiorgan failure with emphasis on acute kidney injury and severity of COVID19: systematic review and meta-analysis. Can J Kidney Heal Dis. (2020) 7:2054358120938573. doi: 10.1177/2054358120938573

3. Wijaya I, Andhika R, Huang I. The use of therapeutic-dose anticoagulation and its effect on mortality in patients with COVID-19: a systematic review. Clin Appl Thromb. (2020) 26:1076029620960797. doi: 10.1177/1076029620960797

4. Vaira LA, Deiana G, Fois AG, Pirina P, Madeddu G, De Vito A, et al. Objective evaluation of anosmia and ageusia in COVID-19 patients: single-center experience on 72 cases. Head Neck. (2020) 42:1252-8. doi: 10.1002/hed.26204

5. De Vito A, Geremia N, Fiore V, Princic E, Babudieri S, Madeddu G. Clinical features, laboratory findings and predictors of death in hospitalized patients with COVID-19 in Sardinia, Italy. Eur Rev Med Pharmacol Sci. (2020) 24:7861-8. doi: 10.26355/eurrev_202007_22291

6. De Vito A, Fiore V, Princic E, Geremia N, Napodano CMP, Muredda AA, et al. Predictors of infection, symptoms development, and mortality in people evidence of using the De Ritis ratio for prognostication in COVID-19.

\section{Implication for Clinical Practice}

Although this "traditional" ratio was initially found in 1957 as a diagnostic test for viral hepatitis (40), it is still commonly used and proves to be a valuable indicator of liver disease (38). It is a promising, straightforward, and readily available parameter for poor prognosis in COVID-19. This meta-analysis showed that AST, but not ALT, was significantly associated with poor prognosis in COVID-19. This supports the use of De Ritis ratio in addition to AST and ALT levels. However, we suggest, including this parameter and other accessible hematological markers, to improve the prognostic performance of the model for COVID19. De Ritis ratio would be better for this marker to be a part of a prognostic model rather than a stand-alone examination. A predictive model comprising of readily available tools may be of value, especially in rural areas where sophisticated prognostic biomarkers are often not available.

In conclusion, elevated De Ritis ratio is associated with poor prognosis in patients with COVID-19.

\section{DATA AVAILABILITY STATEMENT}

The raw data supporting the conclusions of this article will be made available by the authors, without undue reservation.

\section{AUTHOR CONTRIBUTIONS}

RP: conceptualization, methodology, formal analysis, investigation, and writing-original draft. $\mathrm{IH}$ : data curation, investigation, writing-original draft, and project administration. ML: data curation, investigation, and writingoriginal draft. EY and RV: investigation and writing-original draft. AL, SN, BS, and RK: investigation and writing-review and editing. All authors contributed to the article and approved the submitted version.

with SARS-CoV-2 living in retirement nursing homes. PLoS ONE. (2021) 16:e0248009. doi: 10.1371/journal.pone.0248009

7. Zinellu A, Arru F, De Vito A, Sassu A, Valdes G, Scano V, et al. The De Ritis ratio as prognostic biomarker of in-hospital mortality in COVID-19 patients. Eur J Clin Invest. (2021) 51:1-8. doi: 10.1111/eci.13427

8. Huang I, Pranata R, Lim MA, Oehadian A, Alisjahbana B. Creactive protein, procalcitonin, $\mathrm{D}$-dimer, and ferritin in severe coronavirus disease-2019: a meta-analysis. Ther Adv Respir Dis. (2020) 14:175346662093717. doi: 10.1177/1753466620937175

9. Wibowo A, Pranata R, Lim MA, Akbar MR, Martha JW. Endotheliopathy marked by high von Willebrand Factor (vWF) antigen in COVID-19 is associated with poor outcome: a systematic review and meta-analysis. Int $J$ Infect Dis. (2021). doi: 10.1016/j.ijid.2021.06.051

10. Wibowo A, Pranata R, Astuti A, Tiksnadi BB, Martanto E, Martha JW, et al. Left and right ventricular longitudinal strains are associated with poor outcome in COVID-19: a systematic review and meta-analysis. J Intensive Care. (2021) 9:9. doi: 10.1186/s40560-020-00519-3

11. Martha JW, Pranata R, Wibowo A, Lim MA. Tricuspid annular plane systolic excursion (TAPSE) measured by echocardiography and mortality in COVID19: a systematic review and meta-analysis. Int J Infect Dis. (2021) 105:3516. doi: 10.1016/j.ijid.2021.02.029 
12. Wibowo A, Pranata R, Akbar MR, Purnomowati A, Martha JW. Prognostic performance of troponin in COVID-19: a diagnostic meta-analysis and metaregression. Int J Infect Dis. (2021) 105:312-8. doi: 10.1016/j.ijid.2021.02.113

13. Kukla M, Skonieczna-Zydecka K, Kotfis K, Maciejewska D, Łoniewski I, Lara LF, et al. COVID-19, MERS and SARS with concomitant liver injury-systematic review of the existing literature. J Clin Med. (2020) 9:1420. doi: 10.3390/jcm9051420

14. Pranata R, Yonas E, Huang I, Lim MA, Nasution SA, Kuswardhani RAT. Fibrosis-4 index and mortality in coronavirus disease 2019. Eur J Gastroenterol Hepatol. (2021). doi: 10.1097/MEG.0000000000002091

15. Yazar H, Kayacan Y, Ozdin M. De Ritis ratio and biochemical parameters in COVID-19 patients. Arch Physiol Biochem. (2020) 1-5. doi: 10.1080/13813455.2020.1788604

16. Qin C, Wei Y, Lyu X, Zhao B, Feng Y, Li T, et al. High aspartate aminotransferase to alanine aminotransferase ratio on admission as risk factor for poor prognosis in COVID-19 patients. Sci Rep. (2020) 10:16496. doi: 10.1038/s41598-020-73575-2

17. Medetalibeyoglu A, Catma Y, Senkal N, Ormeci A, Cavus B, Kose M, et al. The effect of liver test abnormalities on the prognosis of COVID-19. Ann Hepatol. (2020) 19:614-21. doi: 10.1016/j.aohep.2020.08.068

18. Chen F, Chen W, Chen J, Xu D, Xie W, Wang X, et al. Clinical features and risk factors of COVID-19-associated liver injury and function: a retrospective analysis of 830 cases. Ann Hepatol. (2021) 21:100267. doi: 10.1016/j.aohep.2020.09.011

19. Paliogiannis P, Zinellu A, Scano V, Mulas G, de Riu G, Pascale RM, et al. Laboratory test alterations in patients with COVID-19 and non COVID19 interstitial pneumonia: a preliminary report. J Infect Dev Ctries. (2020) 14:685-90. doi: 10.3855/jidc.12879

20. Benedé-Ubieto R, Estévez-Vázquez O, Flores-Perojo V, et al. Abnormal liver function test in patients infected with coronavirus (SARS-CoV-2): a retrospective single-center study from Spain. J Clin Med. (2021) 10:1039. doi: $10.3390 /$ jcm 10051039

21. Ramos-Lopez O, San-Cristobal R, Martinez-Urbistondo D, et al. Proinflammatory and hepatic features related to morbidity and fatal outcomes in COVID-19 patients. J Clin Med. (2021) 10:3112. doi: 10.3390/jcm10143112

22. Yadlapati S. Prevailing patterns of liver enzymes in patients with COVID-19 infection and association with clinical outcomes. Ann Gastroenterol. (2021). doi: 10.20524/aog.2021.0573. [Epub ahead of print].

23. Andhika R, Huang I, Wijaya I. Severity of COVID-19 in end-stage kidney disease patients on chronic dialysis. Ther Apher Dial. (2020) 25:7069. doi: 10.1111/1744-9987.13597

24. Huang I, Lim MA, Pranata R. Diabetes mellitus is associated with increased mortality and severity of disease in COVID-19 pneumonia - a systematic review, meta-analysis, and meta-regression: diabetes and COVID-19. Diabetes Metab Syndr Clin Res Rev. (2020) 14:395-403. doi: 10.1016/j.dsx.2020.04.018

25. Pranata R, Lim MA, Huang I, Raharjo SB, Lukito AA. Hypertension is associated with increased mortality and severity of disease in COVID-19 pneumonia: a systematic review, meta-analysis and meta-regression. J Renin-Angiotensin-Aldosterone Syst. (2020) 21:147032032092689. doi: 10.1177/1470320320926899

26. Pranata R, Huang I, Lim MA, Wahjoepramono EJ, July J. Impact of cerebrovascular and cardiovascular diseases on mortality and severity of COVID-19-systematic review, metaanalysis, and meta-regression. J Stroke Cerebrovasc Dis. (2020) 29:104949. doi: 10.1016/j.jstrokecerebrovasdis.2020.104949

27. Pranata R, Soeroto AY, Huang I, Lim MA, Santoso P, Permana H, et al. Effect of chronic obstructive pulmonary disease and smoking on the outcome of COVID-19. Int J Tuberc Lung Dis. (2020) 24:83843. doi: $10.5588 /$ ijtld. 20.0278
28. Pranata R, Lim MA, Yonas E, Vania R, Lukito AA, Siswanto BB, et al. Body mass index and outcome in patients with COVID-19: a dose-response metaanalysis. Diabetes Metab. (2021) 47:101178. doi: 10.1016/j.diabet.2020.07. 005

29. Yonas E, Alwi I, Pranata R, Huang I, Lim MA, Gutierrez EJ, et al. Effect of heart failure on the outcome of COVID-19 - a meta analysis and systematic review. Am J Emerg Med. (2020) 46:204-11. doi: 10.1016/j.ajem.2020.07.009

30. Pranata R, Supriyadi R, Huang I, Permana H, Lim MA, Yonas E, et al. The association between chronic kidney disease and new onset renal replacement therapy on the outcome of COVID-19 patients: a meta-analysis. Clin Med Insights Circ Respir Pulm Med. (2020) 14:1179548420959165. doi: 10.1177/1179548420959165

31. Pranata R, Henrina J, Raffaello WM, Lawrensia S, Huang I. Diabetes and COVID-19: the past, the present, and the future. Metabolism. (2021) 121:154814. doi: 10.1016/j.metabol.2021.154814

32. Huang C, Wang Y, Li X, Ren L, Zhao J, Hu Y, et al. Clinical features of patients infected with 2019 novel coronavirus in Wuhan, China. Lancet. (2020) 395:497-506. doi: 10.1016/S0140-6736(20)30183-5

33. Paliogiannis $\mathrm{P}$, Zinellu A. Bilirubin levels in patients with mild and severe Covid-19: a pooled analysis. Liver Int. (2020) 40:1787-8. doi: 10.1111/liv.14477

34. Xu Z, Shi L, Wang Y, Zhang J, Huang L, Zhang C, et al. Pathological findings of COVID-19 associated with acute respiratory distress syndrome. Lancet Respir Med. (2020) 8:420-2. doi: 10.1016/S2213-2600(20)30076-X

35. Alqahtani SA, Schattenberg JM. Liver injury in COVID19: the current evidence. United Eur Gastroenterol J. (2020) 8:509-19. doi: 10.1177/2050640620924157

36. Chai X, Hu L, Zhang Y, Han W, Lu Z, Ke A, et al. Specific ACE2 expression in cholangiocytes may cause liver damage after 2019-nCoV infection. bioRxiv. (2020). doi: 10.1101/2020.02.03.931766

37. Ma C, Cong Y, Zhang H. COVID-19 and the digestive system. Am J Gastroenterol. (2020) 115:1003-6. doi: 10.14309/ajg.0000000000000691

38. Botros M, Sikaris KA. The de ritis ratio: the test of time. Clin Biochem Rev. (2013) 34:117-30.

39. Safari R, Gholizadeh P, Marofi P, Zeinalzadeh E, Pagliano P, Ganbarov K, et al. Alteration of liver biomarkers in patients with SARS-CoV-2 (COVID-19). J Inflamm Res. (2020) 13:285-92. doi: 10.2147/JIR.S257078

40. De Ritis F, Coltorti M, Giusti G. An enzymic test for the diagnosis of viral hepatitis: the transaminase serum activities. Clin Chim Acta. (1957) 2:70-4. doi: 10.1016/0009-8981(57)90027-X

Conflict of Interest: The authors declare that the research was conducted in the absence of any commercial or financial relationships that could be construed as a potential conflict of interest.

Publisher's Note: All claims expressed in this article are solely those of the authors and do not necessarily represent those of their affiliated organizations, or those of the publisher, the editors and the reviewers. Any product that may be evaluated in this article, or claim that may be made by its manufacturer, is not guaranteed or endorsed by the publisher.

Copyright (c) 2021 Pranata, Huang, Lim, Yonas, Vania, Lukito, Nasution, Siswanto and Kuswardhani. This is an open-access article distributed under the terms of the Creative Commons Attribution License (CC BY). The use, distribution or reproduction in other forums is permitted, provided the original author(s) and the copyright owner(s) are credited and that the original publication in this journal is cited, in accordance with accepted academic practice. No use, distribution or reproduction is permitted which does not comply with these terms. 